\title{
Coherence between radar observations of magnetospheric field line resonances and discrete oscillations in the solar wind
}

\author{
J. A. E. Stephenson and A. D. M. Walker \\ School of Physics, University of KwaZulu-Natal, Private Bag x54001, Durban, 4000, South Africa
}

Received: 6 November 2009 - Accepted: 17 December 2009 - Published: 13 January 2010

\begin{abstract}
Field line resonances have been observed for decades by ground-based and in situ instruments. The driving mechanism(s) are still unclear, although previous work has provided strong grounds that coherent waves in the solar wind may be a source. Here we present further evidence, with the use of multitaper analysis, a sophisticated spectrum estimation technique. A set of windows (dpss tapers) is chosen with characteristics that best suit the width of the narrowband peaks to be identified. The orthogonality of the windows allows for a confidence level (of say 95\%) against a null hypothesis of a noisy spectrum, so that significant peaks can be identified. Employing multitaper analysis we can determine the phase and amplitude coherence at the sampling rate of the data sets and, over their entire duration. These characteristics make this technique superior to single windowing or wavelet analysis. A high degree of phase and amplitude (greater then 95\%) coherence is demonstrated between a $2.1 \mathrm{mHz}$ field line resonance observed by the SHARE radar at Sanae, Antarctica and the solar wind oscillation detected by WIND and ACE satellites.
\end{abstract}

Keywords. Magnetospheric physics (Solar windmagnetosphere interactions)

\section{Introduction}

ULF pulsations have been observed for many years in magnetometer, radar and other data. The existence of narrow band resonances is now well established. Those in the Pc5 band (period 150 to $600 \mathrm{~s}$ ) are global magnetohydrodynamic events in the magnetosphere. There are two main types. We focus here on observations of Field Line Resonances (FLRs) for which a magnetic shell, defined by a particular value of

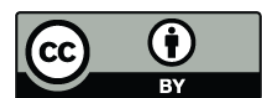

Correspondence to: J. A. E. Stephenson (stephens@ukzn.ac.za)
$L$, its equatorial radius, has a natural frequency of oscillation, determined by the Alfvén velocity and field line length. If some external influence having a well defined frequency excites the magnetosphere into oscillation then energy is transferred deep into the magnetosphere through the fast mode. The $L$-shell with frequencies near the driving frequency will resonate and oscillations will be set up over a narrow set of latitudes.

The source of the oscillations driving field line resonances (FLRs) in the magnetosphere remains controversial. Several mechanisms have been proposed, the earliest being a Kelvin-Helmholtz instability on the magnetopause (Southwood, 1968). However, this could not explain discrete field line resonances occurring simultaneously at different latitudes. These have been attributed to cavity or waveguide modes in the region between the magnetosphere and a reflection level within it (Kivelson and Southwood, 1985). The bounded magnetosphere establishes eigenmodes with discrete frequncies, each of which leaks evanescently to a FLR at a latitude having the same natural frequency. The excitation of the waveguide modes is assumed to be associated with processes at, or outside, the magnetopause. Such a process might be associated with over-reflection at the magnetopause by a mechanism such as that described by Mann et al. (1999). Another possibility is that a source external to the magnetosphere could drive the resonance. One such source could be a broad band impulse from the solar wind exciting the cavity modes in the magnetosphere at their natural frequencies. Another is a coherent oscillation originating from an upstream source in the solar wind. Coherent MHD waves have been observed by a number of authors in the solar wind as described by the review of Barnes (1979) and references therein.

Thomson et al. (1995) took 118 optically observed $p$ mode frequencies between 1.5 and $2.4 \mathrm{mHz}$ and compared them to the group frequencies from solar wind particle data observed by Ulysses. Helioseismic oscillations of the Sun are acoustic 
phenomena, driven by pressure ( $p$ modes) or by gravity ( $g$ modes) gradients. The frequencies of the $p$ modes have been mapped thoroughly using optical techniques (Libbrecht et al., 1990) in the Pc5 band. The power spectral density is quite similar to that of FLRs. However, correlation of optical peaks with groundbased observations will always be successful since helioseismic modes are much more stable than FLRs, which means that their spectral resolution is of the order of $\mu \mathrm{Hz}$. In contrast, FLRs are relatively short lived, so spectral analysis typically cannot provide resolution better then about $0.1 \mathrm{mHz}$. On the other hand, correlations between ULF pulsations in the solar wind (at frequencies very similar to $p$ modes) with ground-based radar data are more meaningful. The match between frequency estimates was good and the particle spectrum was found to be dominated by individual spectral lines. This implies that charged particles are being scattered, and therefore diffused, in the interplanetary medium not only by a continuous spectrum of IMF fluctuations but also by discrete wave frequencies. Other works (Thomson et al., 2001, 2002; Ghosch et al., 2009) have strengthened the case that $p$ modes excite waves in the solar wind.

Statistical studies (Baker et al., 2003) of magnetometer data exhibit no preference for narrow band resonances to occur at particular frequencies. However, individual observations of resonances do show narrow spectral peaks. In particular, visual inspections of Doppler velocity observations of FLRs in radar data reveals that these pulsations are stable over many hours. In addition, they tend to occur near 1.3, 1.9, 2.7 and $3.3 \mathrm{mHz}$ (Fenrich and Samson, 1995; Ruohoniemi et al., 1991; Samson et al., 1992; Walker et al., 1992). Observations of monochromatic FLRs in the Pc5 range are usually attributed to global cavity (Kivelson and Southwood, 1985) or waveguide (Samson et al., 1992) modes. This would require a cavity of constant dimensions and unvarying physical properties, inconsistent with a dynamic magnetosphere. A model, which can explain qualitatively the occurrence of discrete resonances at the preferred frequencies by assuming they arise from discrete monochromatic MHD waves incident on the bow shock from the upstream solar wind, has been provided by Walker $(2002,2005)$. This assumption requires an unspecified mechanism generating these waves. Coherent waves are propagated through the bow shock into the magnetosheath. In general the wave is reflected from the magnetopause. At some points, however, the frequency of the incident wave matches the natural frequency of the cavity oscillation which is then strongly excited. Beyond the turning point that bounds the cavity, the FLR is excited.

We have previously presented HF radar observations of a Pc5 FLR associated with oscillations seen in the solar wind by instruments aboard the WIND spacecraft (Stephenson and Walker, 2002). In addition, Eriksson et al. (2005) have reported correlations between HF radar observations of FLRs and solar wind pressure oscillations. Similar observations have been made by Kepko et al. (2002) relat- ing oscillations in the solar wind to pulsations at geosynchronous orbit. Other authors (Potemra et al., 1989; Shimazu et al., 1995; Prikryl et al., 1998, 1999) have also reported ground-based observations of quasi-periodic phenomena associated with solar wind oscillations. There have also been observations of surface waves on the magnetopause correlated with solar wind oscillations at discrete frequencies (Sibeck, 1992). More recently, (Fenrich and Waters, 2008) have demonstrated a high degree of phase coherence between ACE density and Kodiak HF radar observations utilizing FFT and wavelet cross-phase measurements.

In this paper we present a detailed analysis of HF radar observations of FLRs arising from magnetospheric cavity or waveguide compressional modes, associated with solar wind MHD waves. We quantify their coherence using a multitaper analysis. This technique is most efficient when applied to a long (with respect to the period $1-5 \mathrm{mHz}$ pulsation band), continuous data series. Coherent scatter is a condition for reflection of the radar signal implying that long intervals of good quality radar data are not very common. Data from the SHARE HF radar at Sanae, Antarctica on the 7 June 2000 were selected as they exhibited a very sustained period (01:31 UT-19:00 UT) of clear FLR signature. We have analyzed several other events of 7 May 2005 and 28 May 2006 which show similar features. We focus on a resonance near $2.1 \mathrm{mHz}$ common to both radar and satellite data sets in sections concerning details of the methodology of our analysis. Section 4 is divided into four subsections. The first two sub-sections concern the motivation and method of the multitaper approach. Following that, in Sect. 4.3, the procedure by which a reconstruction of significant narrowband signals, by complex demodulation from the mutitaper spectrum, is explained. The reconstruction is compared to a signal reconstructed from a spectrum calculated with an application of a single Hanning window. Included in this sub-section is a description of the equations required when calculating the energy flux of the $2.1 \mathrm{mHz}$ resonance in the solar wind and FLR in the magnetosphere. Finally, in Sect. 4.4, a description and presentation of coherence between the solar wind and radar data, using efficient multitaper methods are presented.

\section{Satellite observations}

For the FLR event under investigation the WIND satellite was at $(55,-40,-10) R_{\mathrm{E}} \mathrm{GSE}$. The velocity, magnetic field components, proton temperature and number density $60 \mathrm{~s}$ key parameters from the SWE and MFI (Lepping et al., 1995; Ogilvie et al., 1995) experiments are plotted in Fig. 1. Data were also used from the ACE satellite, which orbits near to the L1 libration point. Its GSE coordinates for this event were $(235,40,-15) R_{\mathrm{E}}$. The two spacecraft were separated by some distance, ACE being around $180 R_{\mathrm{E}}$ upstream of WIND. Level $264 \mathrm{~s}$ velocity, magnetic, thermal velocity and number density data were used. The ACE solar wind 


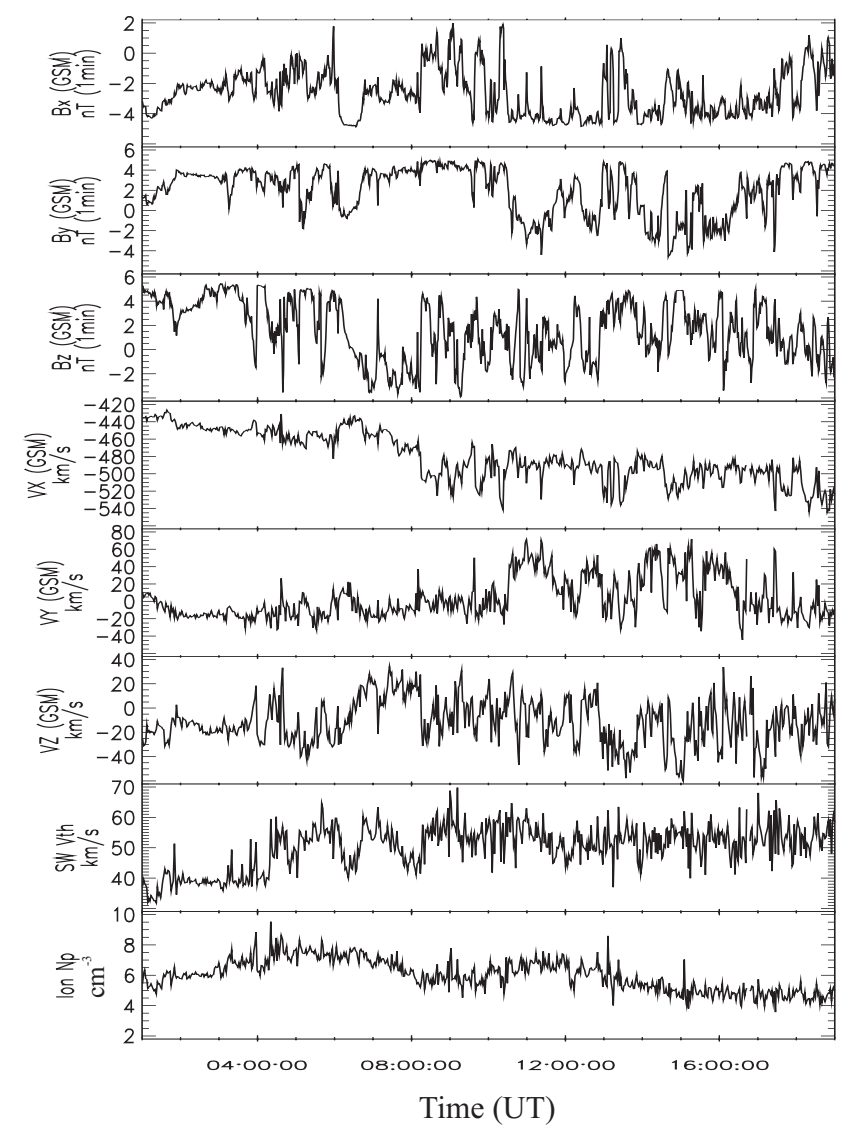

Fig. 1. WIND magnetic field, velocity, thermal velocity and number density observations for 7 June 2000.

parameters from the SWEPAM (McComas et al., 1998) and MFI (Smith, 1998) experiments, in GSM coordinates, are plotted in Fig. 2.

All parameters plotted here were subjected to spectral analysis and subsequent complex demodulation. Satellite data were linearly interpolated to fill in small data gaps.

The reader's attention is drawn to the particularly long duration of this event, lasting over $17 \mathrm{~h}$. This case study was characterized by relatively quiet magnetic conditions with the total magnetic field less than $8 \mathrm{nT}$. The average solar wind velocity and density values were typical for the solar wind with values of around $480 \mathrm{~km} \mathrm{~s}^{-1}$ and $6 \mathrm{~cm}^{-3}$. Of particular interest here is a commencement of significant oscillatory behaviour in all solar wind parameters in Figs. 1 and 2, coincidental with the onset of FLRs in the radar data.

\section{Radar observations}

The radar data were from the SHARE radar (Dudeney et al., 1994) at Sanae, Antarctica. The field of view of this radar is illustrated in Fig. 3. The SHARE radar forms part of

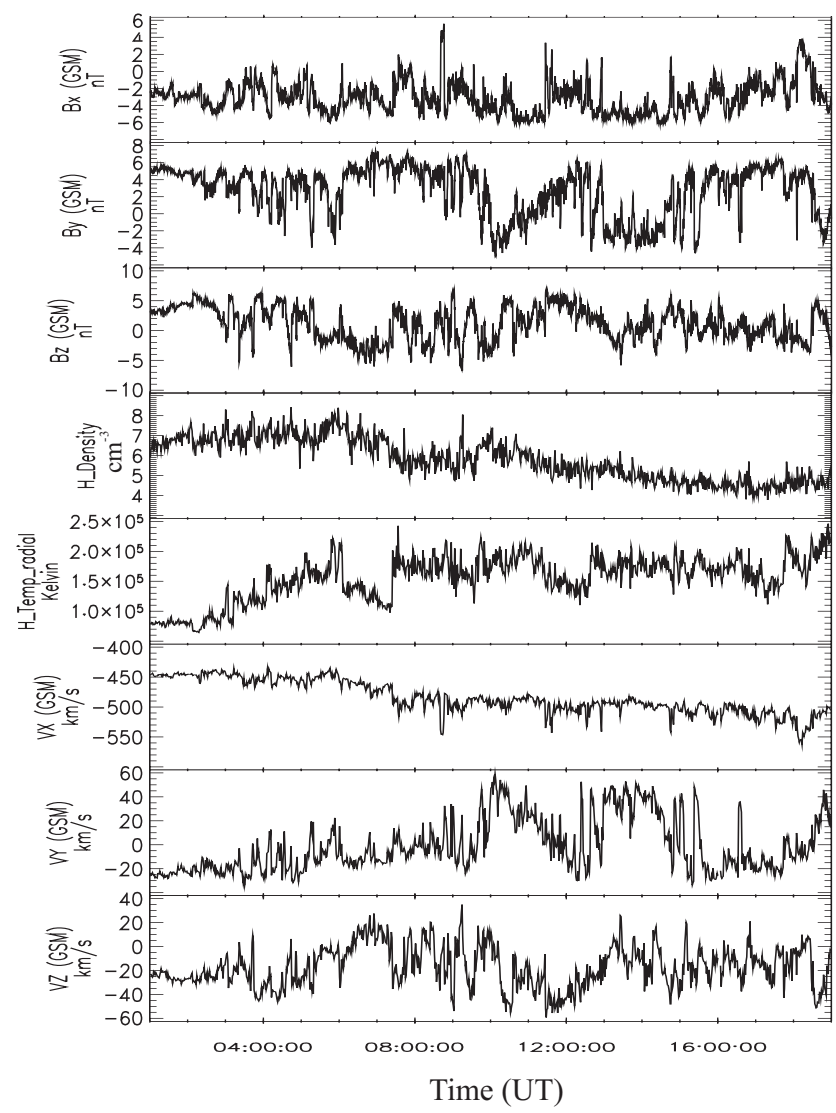

Fig. 2. ACE magnetic field, number density, proton temperature and velocity parameters for 7 June 2000 .

the international SuperDARN network of radars (Chisham et al., 2007). Such coherent radars provide measurements of backscattered power, Doppler velocity and spectral width of field aligned irregularities in the ionosphere. The SHARE radar used $16 \log$ periodic antennas operating in the 8$20 \mathrm{MHz}$ band. These were replaced in 2009 by a new design. The radar's field of view is divided into 16 beams which, in normal mode, are stepped through one at a time by means of a phasing matrix. The time resolution of one scan is typically one to two minutes, depending on the mode of operation. In the event presented here the SHARE radar was operating in a normal mode i.e. stepping through each beam one at a time for an integration time that corresponds to a $120 \mathrm{~s}$ scan. The beam separation is a few degrees (depending on the operating frequency), giving a total azimuthal field of view of about $52^{\circ}$. Depending on favourable scattering conditions, reflection can be obtained from up to 75 range gates, spaced $45 \mathrm{~km}$ apart. This "fan" of beams covers very large areas of up to $2000 \times 2000 \mathrm{~km}^{2}$. This large spatial coverage makes HF radars ideal tools for resolving the structure of FLRs.

The ULF oscillation in the F region plasma flows, associated with FLRs, are observable in the Doppler velocities 


\section{SANAE HF RADAR FOV}
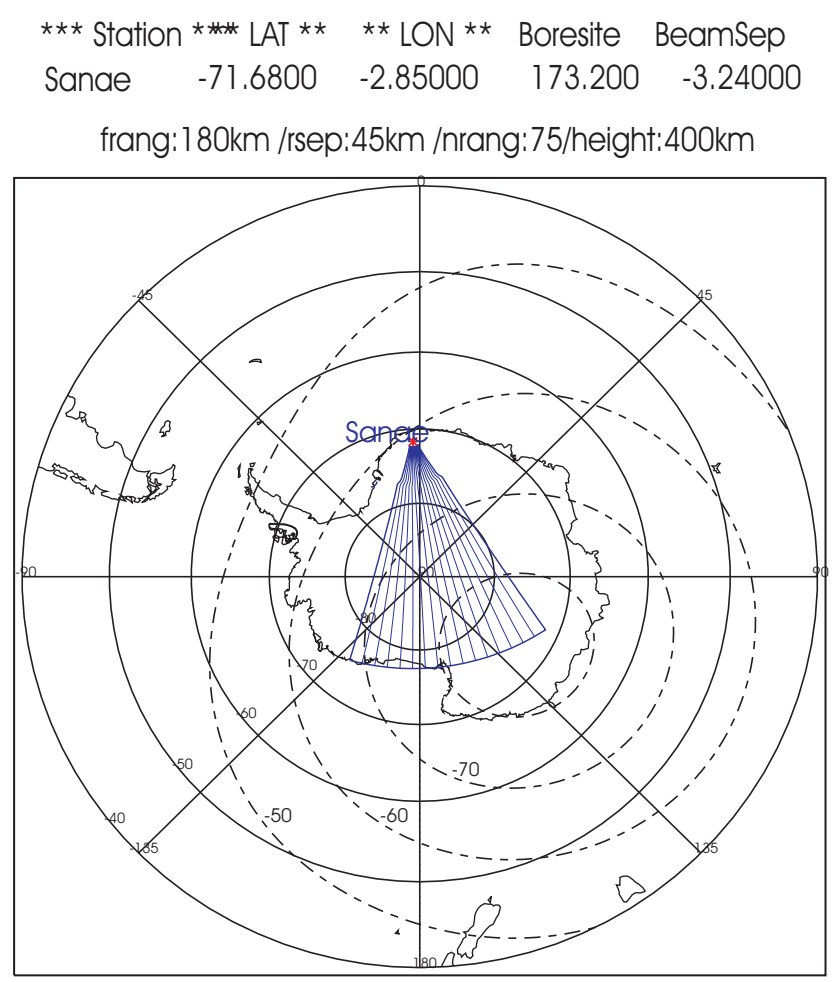

Fig. 3. The SHARE radar field of view in geographic coordinates. Lines of magnetic latitude (dashed) are also shown.

of each beam. FLR maxima are polarized with east-west velocity. For this reason, data from the beam most closely aligned to $L$-shells (shown as broken lines in Fig. 3) were selected for analysis. This is beam 4 for the SHARE radar. Beams are numbered 0 to 15 from west to east in Fig. 3. A range-time summary plot of line-of-sight Doppler velocity from this beam is presented in the upper panel of Fig. 4 in which the alternating bands of positive and negative velocity characteristic of pulsations is evident. In the lower panel of this figure, a four hour subset (08:00-12:00 UT) of the upper panel is plotted in the form of a stack plot of Doppler velocities over range gates 6 to 23 (corresponding to 450 to $1215 \mathrm{~km}$ ). Careful inspection of this panel reveals that for example, near 09:30 UT, pulsation amplitudes maximize over a narrow set of ranges corresponding to geomagnetic latitudes around $66^{\circ} \mathrm{S}$. In addition, there is a phase shift of approximately 180 degrees in the pulsation activity with latitude through the maximum resonance amplitude. These characteristics are predicted by resonance theory, and they are peculiar to FLRs as opposed to any other type of standing wave.

\section{Data analysis}

\subsection{Thomson's multitaper method of spectral analysis}

When Fourier analyzing data series of finite length it is usual practice to window the data. This avoids sharp discontinuities and the related problems they produce in spectral estimation. Multiple windowing (tapering) of data has several advantages over single windowing, for which the data time series is effectively reduced in a trade-off to reduce bias due to leakage. Multitaper estimates arise from the inverse theory solution of the integral equation relating the Fourier transform of the available data to that of the ideal complete sample (Thomson, 1982). We use the windows described by Thomson. They are the discrete prolate spheriodal sequences (dpss) that are the eigenfunctions of the kernel of the integral equation. They are mutually orthogonal. Each window covers different parts of the data series. The multiple windows are therefore collectively more efficient than any unique window.

When choosing the number of windows to be applied to a sample of $N$ equally spaced data $x(t)$, we must compromise between statistical stability and frequency resolution (Percival and Walden, 1993). A time bandwidth product of $N W \triangle t=5$ ( $W$ is the half bandwidth) is used here. The bandwidth choice was principally determined by the spectral width of the FLRs, typically a fraction of a $\mathrm{mHz}$. Eight ( $K \leq 2 N W \triangle t=8$, a condition for tapers with good first moment properties) tapers $\left(v_{t}{ }^{k}(N, W)\right)$ were applied. Maximizing the number of tapers, while reducing bias more effectively, implies a larger convolution bandwidth, which could smooth out the peaks of interest. The windowed Fourier transforms, or eigenspectra, are:

$X_{k}(f)=\sum_{t=0}^{N-1} e^{-i 2 \pi f t} v_{t}^{k}(N, W) x(t)$

The multitaper estimate is then

$S(f)=\sum_{k=0}^{K-1}\left|d_{k}(f) X_{k}(f)\right|^{2}$

$d_{k}$ are weights. Discussion of their determination can be found in Thomson (1982).

The multitaper method offers the appeal of being nonparametric in that it does not prescribe an a priori model for the process generating the time series under analysis. The statistical properties of this spectrum estimate have been carefully studied. Relevant conclusions for this work include the following points (Percival and Walden, 1993).

1. The spectral resolution of a multitaper estimate is $2 \mathrm{~W}$.

2. The eigenspectrum determined from each taper is approximately distributed as a chi-square random variable with two degrees of freedom (Brillinger, 1981). And, 


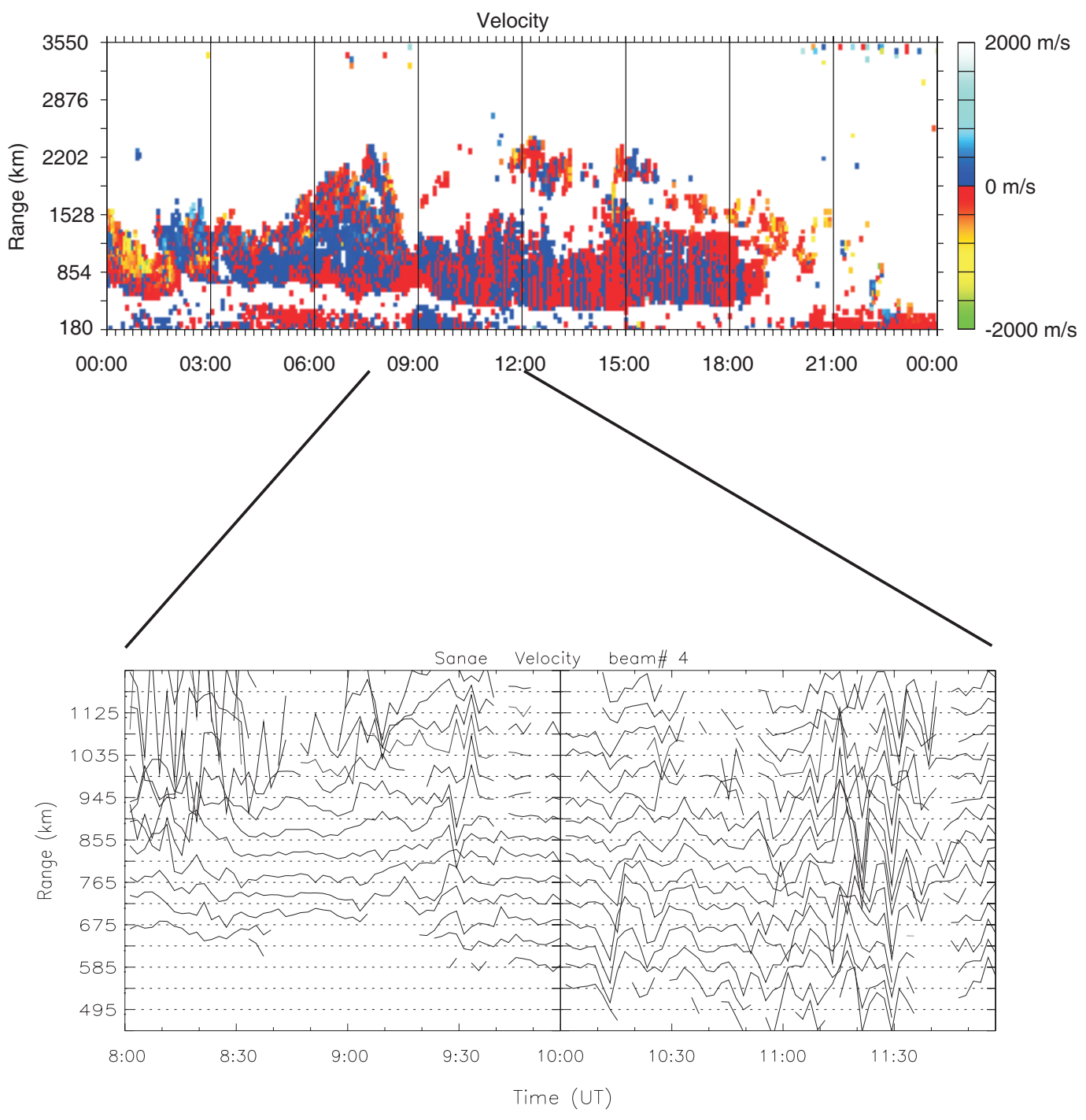

Fig. 4. SHARE range-time velocity plot for 7 June 2000. A four hour subset of beam 4 Doppler velocity is plotted to illustrate FLR activity.

since each taper is independent, the multi-taper spectrum follows a chi-square distribution with $2 K$ degrees of freedom. Using this assumption, a detection threshold (say 95\% level) may be set against a null hypothesis of a noisy spectrum (Thomson, 1982). This implies that a modest increase in taper numbers are enough to noticeably shrink the confidence interval.

3. In addition, a non-parametric variance of the multi-taper spectrum may be calculated by "jack-knifing", which is achieved by deleting each window in turn from the analysis.

\subsection{Multitaper spectra of solar wind and radar data}

The SSA-MTM Toolkit (Dettinger et al., 1995; Ghil et al., 2002) was used to perform multitaper spectral estimates of the solar wind data and SHARE Doppler velocity data.
The multitaper spectra of concurrent ACE solar wind parameters and radar observations (range gate 15) were computed and plotted in Fig. 5. The two most significant peaks in the radar data have been shaded to illustrate coincidences with ACE data. The dashed lines associated with the spectra are levels of $95 \%$ significance. From inspection of Fig. 5 we can see that the $1.9 \mathrm{mHz}$ pulsation is present above the $95 \%$ significance in all the ACE components whereas the $2.1 \mathrm{mHz}$ resonance achieves this level in 5 of the $8 \mathrm{ACE}$ components. The $2.1 \mathrm{mHz}$ narrowband peak is the most significant FLR and so it was selected for more detailed analysis described below and in Sects. 4.3 and 4.4.

The spectra (calculated for a time bandwidth product $N W \triangle t=5$ ) for range gates 13 to 17 are stacked in ascending order (13 at the bottom) in Fig. 6. Each power spectral density spectrum is plotted together with a $95 \%$ confidence level. Power is plotted on a log scale as this implies 


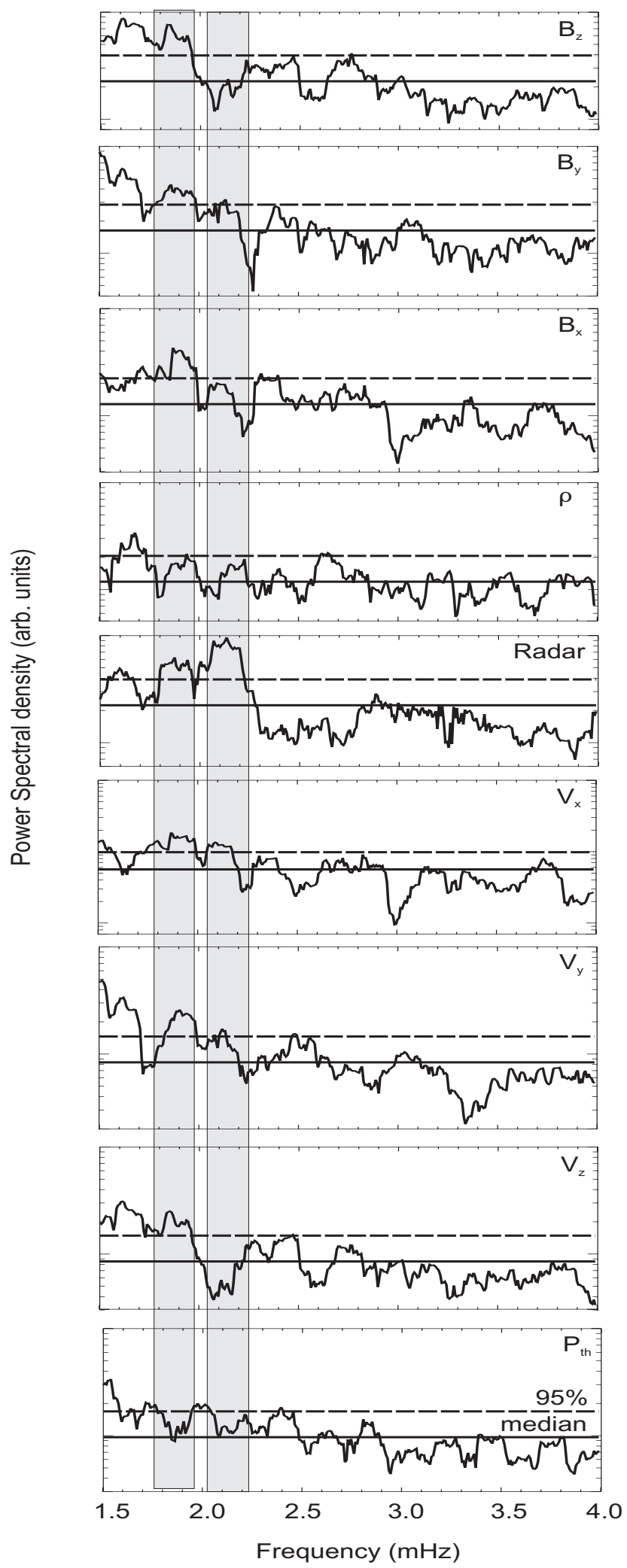

Fig. 5. Multitaper spectra $(N W \triangle t=5 ; K=8)$ of ACE components (01:00-20:00 UT) and radar Doppler velocity for beam 4 gate 15 (02:31-19:00 UT) on 7 June 2000.

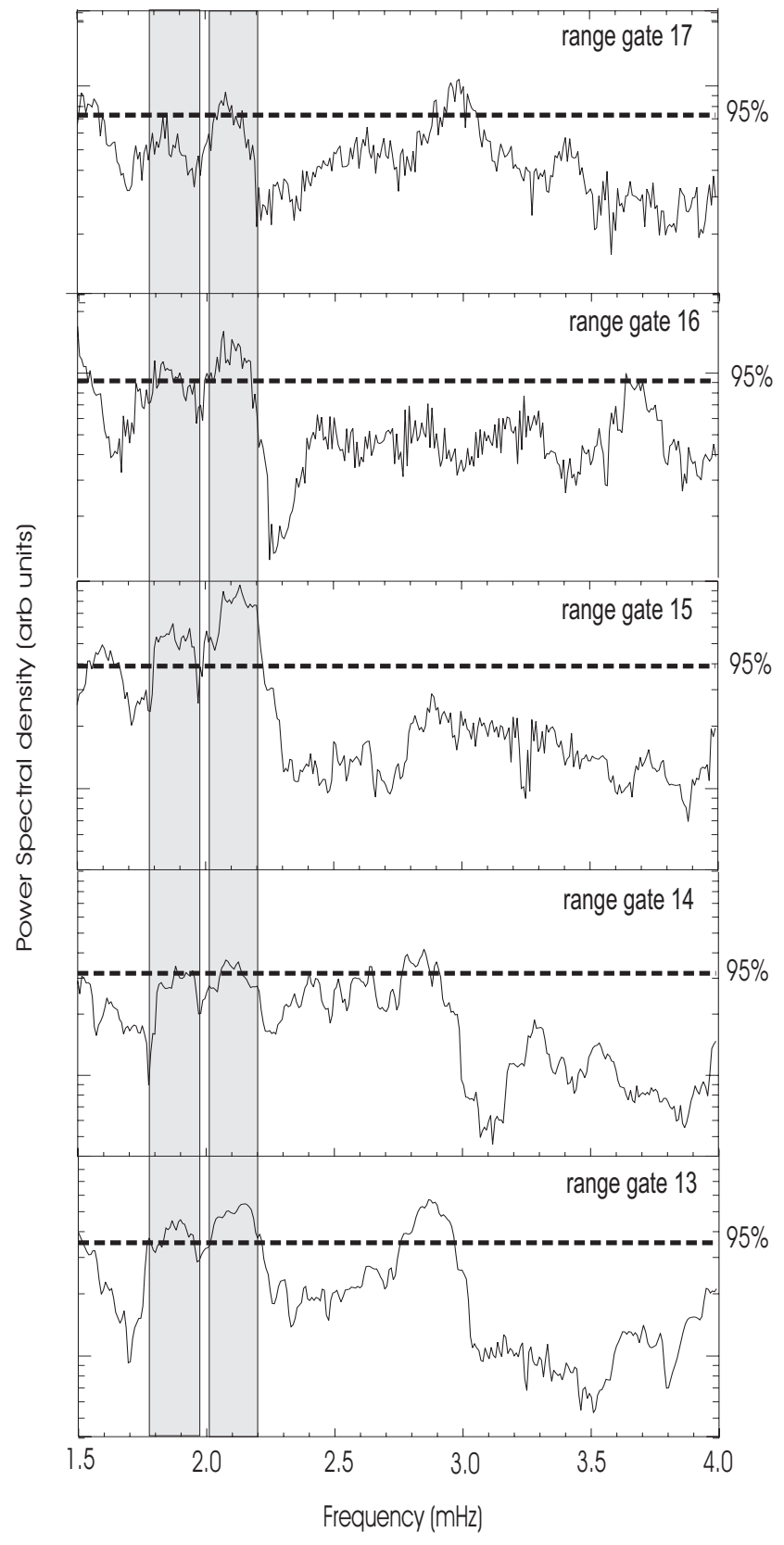

Fig. 6. Multitaper spectra $(N W \triangle t=5 ; K=8)$ of radar Doppler velocity beam 4 range gates $13-17$ on 7 June 2000 . Data intervals for each gate are variable depending on data availability.

a confidence interval independent of frequency. The spectral resolution $(2 \mathrm{~W})$ of the higher range gates is better than that of the lower ones as these range gates had longer periods of continuous data. An upper limit of half bandwidth $(W)$ was set $0.15 \mathrm{mHz}$ for range gate 10 , although for higher range gates this was considerably smaller. For example, tapers applied to range gate 15 data had $W$ of $0.08 \mathrm{mHz}$. This spectrum exhibits two peaks above the $99 \%$ significance level near 1.9 
and $2.1 \mathrm{mHz}$ above a white noise level. These are the most significant peaks in the radar data. There are other peaks that exceed the $95 \%$ significance in other range gates. These are near 1.7 and $2.8 \mathrm{mHz}$.

\subsection{Reconstruction by complex demodulation}

The amplitude and phase of the narrowband resonances were determined by a method of complex demodulation whereby the data were bandpass filtered and an analytic signal was determined. The analytic signal $f(t)$ may be regarded as an objective estimate of the instantaneous amplitude and phase of the quasi-monochromatic signal $F(t)$ and is calculated from

$$
f(t)=F(t)-i \operatorname{Hi}(t)
$$

where $\mathrm{Hi}(t)$ is the Hilbert transform of $F(t)$ (Bracewell, 1986).

As an example, the $2.1 \mathrm{mHz}$ band analytic signal of the radar Doppler velocity for beam 4 and gate 15 is plotted together with the raw velocity values in Figs. 7a and b. Data from range gate 15 were analysed since, from inspection of the stack plot of radar spectra of Fig. 6, this range exhibited a maximum amplitude of the $2.1 \mathrm{mHz}$ resonance. Smaller, but significant peaks are visible in adjacent range gates. The green analytic envelope in Fig. $7 \mathrm{~b}$ is a reconstruction of the 99\% significance narrowband peak from a multitaper spectrum. The bandpass filter width is $2 W(0.168 \mathrm{mHz})$ which is effectively the frequency resolution of the multitaper spectrum, as determined by the choice of time bandwidth product $(N W \triangle t=5)$. An analytic signal determined from a cosine Hanning window Fourier spectrum with an equivalent bandpass filter width is plotted on the same axis. Its amplitude is plotted as the black curve that encapsulates the analytic signal determined from this single window. The windowing comparison in this figure demonstrates the efficiency of the multitaper method. Each orthogonal taper windows a different part of the data set. Lower order tapers tend to attenuate the beginning and ending of the data set, whereas higher order tapers pick up this information. The consequence of this is that the multitaper envelope has amplitude and phase information for the duration of the data set whereas that determined from a single window is attenuated at both ends of the data set. This implies that the resonances that occur in the middle of the data set are the only ones that are not attenuated. In particular, we can see that the second wave packet maximum is severely attenuated by a simple Hanning window. Comparison of Figs. 7a and b clearly demonstrates that there is a maximum in amplitude of the multitaper wave packets whenever strong oscillations are visible in the raw data set, giving confidence that the multitaper method is a more accurate reflection of the $2.1 \mathrm{mHz}$ resonance.
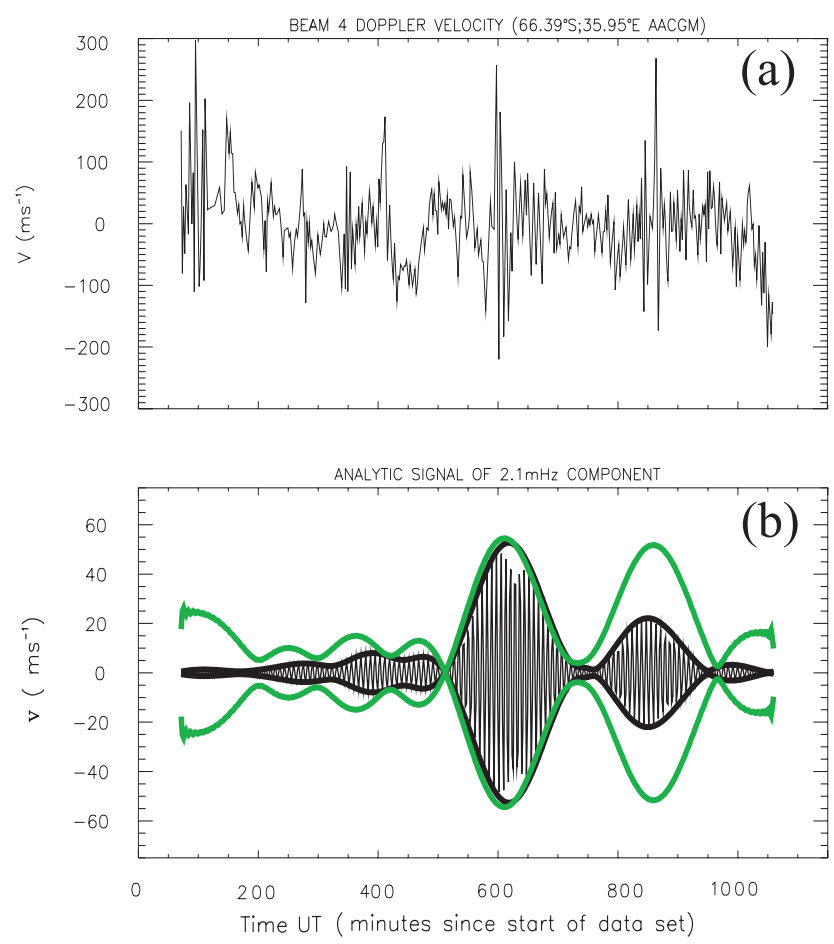

Fig. 7. (a) Beam 4, gate 15 Doppler velocity. (b) Black curve: analytic signal and envelope of reconstructed signal using Hanning window. Green curve: envelope of reconstructed signal using multitaper method.

\subsubsection{Energy flux of reconstructed MHD waves in the solar wind}

Coherent waves in the solar wind are generally thought to be transverse Alfvén or fast waves. Slow waves originating near the Sun would be rapidly damped. MHD waves in the solar wind are anisotropic but nondispersive so that wave velocity is independent of frequency but depends on direction of propagation.

The mean energy flux $(\boldsymbol{S})$ associated with a harmonic MHD wave, varying as $e^{-i \omega t}$, in the solar wind that is incident on a boundary such as the magnetopause is given as (Walker, 2000):

$\boldsymbol{S}=\frac{1}{2} \Re\left\{\left(\mathrm{p}+\frac{\boldsymbol{B} \cdot b}{\mu_{0}}\right) \boldsymbol{v}^{*}-\frac{\boldsymbol{B}}{\mu_{0}} \boldsymbol{b} \cdot v^{*}+U \boldsymbol{V}\right\}$

where

$U=\frac{1}{4}\left\{\frac{\boldsymbol{b} \cdot b^{*}}{\mu_{0}}+\rho_{0} \boldsymbol{v} \cdot \boldsymbol{v}^{*}+\frac{\mathrm{pp}^{*}}{\rho_{0} V_{\mathrm{s}}^{2}}\right\}$

And $\boldsymbol{b}$ is the magnetic field, $\boldsymbol{v}$ is the velocity, $p$ the thermal pressure (calculated with adiabatic assumption), $\rho_{0}$ is the average density and $V_{\mathrm{s}}$ is the speed of sound. $U$ is the energy density which is made up of magnetic, kinetic and adiabatic compression components. The energy flux equation 


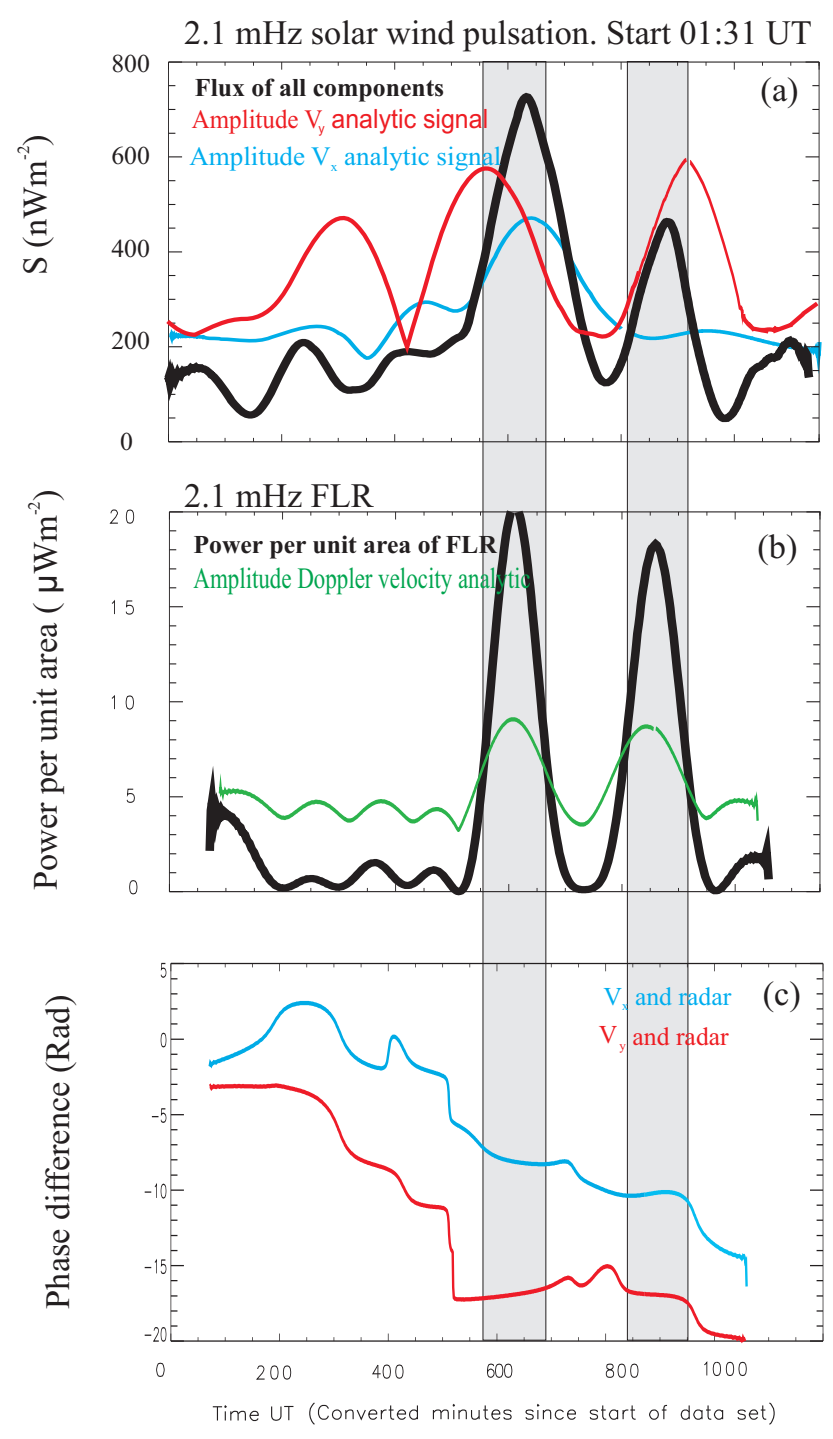

Fig. 8. (a) Amplitude of $2.1 \mathrm{mHz}$ solar wind velocity $\mathrm{x}$ and $\mathrm{y}$ components and energy flux of MHD wave in the solar wind. (b) $2.1 \mathrm{mHz}$ analytic signal and power power unit area of FLR. Start time 01:33 UT, time adjusted by $57 \mathrm{~min}$ in (a) and (b). (c) Cross phase of $2.1 \mathrm{mHz}$ ACE Vx and radar analytic signals (blue) and ACE Vy and radar analytic signals (red).

(Eq. 4) is made up of three terms. There first two terms arising from the MHD waves in the rest frame of the plasma and the third corresponds to the convection of the internal energy density of the solar wind. The mean energy flux therefore depends on the solar wind velocity, changes in solar wind velocity and wave amplitudes. The bold upper case symbols are zero order quantities determined by filtering out the higher frequency components of the relevant data parameter. The lower case symbols are first order perturbations with asterisk denoting their complex conjugate. The first order perturbations, representing MHD waves are reconstructions from the multitaper spectra of each of the solar wind parameters $\left(B_{\mathrm{X}}\right.$, $\left.B_{\mathrm{y}}, B_{\mathrm{z}}, V_{\mathrm{x}}, V_{\mathrm{y}}, V_{\mathrm{z}}, P\right)$. A reconstruction of density spectrum peaks is not necessary due to our adiabatic assumption, thermal pressure is a proxy for density. The multitaper reconstruction of the $2.1 \mathrm{mHz}$ peaks of the ACE $V_{\mathrm{x}}$ and $V_{\mathrm{y}}$ parameters are plotted as blue and red lines in Fig. 8a. The amplitude of the reconstructions of the $2.1 \mathrm{mHz}$ band for all ACE parameters plotted in Fig. 5 were substituted into Eqs. (4) and (5) resulting in the evaluation of the mean energy flux $(S)$ at the front of the magnetopause plotted as the bold line in Fig. 8a. The power density of the $2.1 \mathrm{mHz}$ FLR, calculation described in Sect. 4.3.2, is plotted as the bold curve in Fig. 8b. It has been shifted in order that its maxima coincide with the timing of the $2.1 \mathrm{mHz}$ energy flux maxima measured by the ACE satellite. We can estimate this delay time from the average solar wind velocity with the following reasoning. The energy flux vector is in the same direction as the group velocity. The first two terms between braces in Eq. (4) represent the group velocity $V_{g, 0}$ in the rest frame of the solar wind. The last term is the modification in the frame at rest with respect to the instruments of ACE and WIND satellites. Because $V \gg V_{\mathrm{g}, 0}$ the net energy flux vector $S$ and the group velocity in the observing frame make a relatively small angle with the Sun-Earth line. The delay was 57 min (compared with $55 \mathrm{~min}$ corresponding to the average solar wind velocity), implying the disturbance had travelled at a slightly different speed than the average solar wind velocity as one would expect if the MHD wave has a small velocity relative to the solar wind.

\subsubsection{Energy deposited in the ionosphere by FLRs}

Energy is dissipated in the ionosphere by Joule heating at a rate $\boldsymbol{j} \times \boldsymbol{E}$ in the ionospheric rest frame. For an applied electric field, energy is dissipated by current parallel to the electric field and Joule heating can therefore be estimated as $\Sigma_{p}|E|^{2}$ where $\Sigma_{p}$ is the Pedersen conductivity, assumed to be $5 \Omega^{-1}$ (Greenwald and Walker, 1980). The electric field was calculated from the amplitude of $2.1 \mathrm{mHz}$ multitaper Doppler velocity analytic signal, plotted as the green curve in the second panel Fig. 8b, and the World Magnetic Model 2005 magnetic field value at the coordinates of observation at $300 \mathrm{~km}$ altitude. The mean power per unit area values calculated by this method are shown as the bold curve in Fig. 8b. This curve gives the evolution of the $2.1 \mathrm{mHz}$ FLR for the entire data set at a high temporal resolution which is dependent on the choice of time bandwidth product.

\subsection{Phase and amplitude coherence of solar wind and radar data}

If solar wind oscillations drive FLRs then they should exhibit coherence in both phase and amplitude during an event. Multiple windowing is an efficient method for calculating coherence (Thomson, 1982). Coherence is a measure of the 


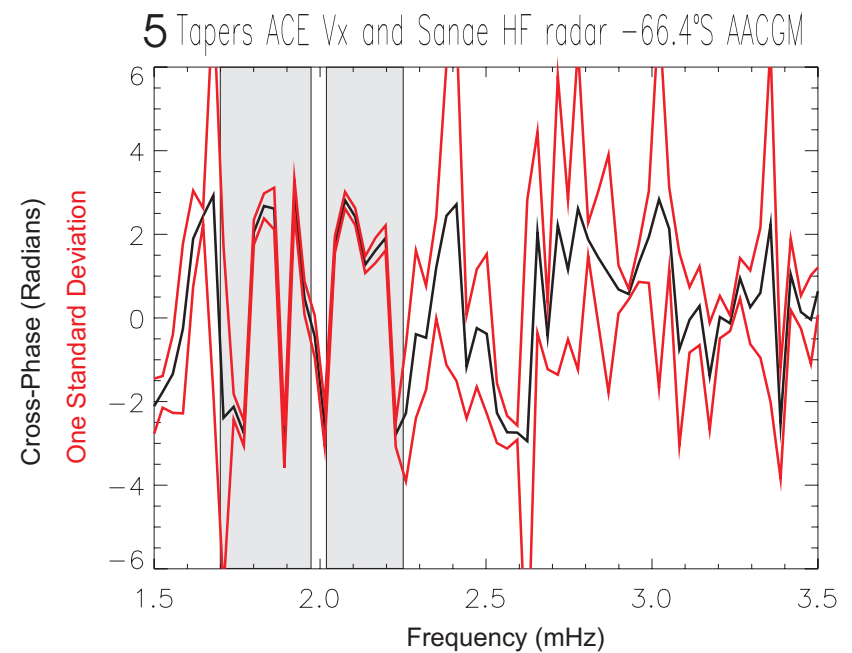

Fig. 9. Phase coherence between ACE Vx and radar Doppler velocity parameters. Cross-phase (phase difference) is plotted as solid line. Red curves are one jack-knife standard deviation on either side of the phase coherence.

association between two time series in the frequency domain. Fundamentally, it is what one obtains by passing each series through a narrow band filter centred at a given frequency, then cross-correlating their outputs.

In order to determine the coherence between ACE and $\mathrm{HF}$ radar measurements, an interpolation of the radar data was performed such that both data had $N=927$ observations with a sampling resolution of $64 \mathrm{~s}$. Increasing the sampling rate of the radar data (from 120 s) is allowable since the frequencies of interest here are much less than the sampling rate as so the spectrum is unaffected in this band. In Sect. 4.1, we described the criterion that we used to select the time-bandwidth product $(N W \triangle t=5)$ and number $(K=8)$ of tapers. We also described the method of their application (Eqs. 1 and 2) in order to obtain a multitaper spectrum of a data series $x(t)$. The cross-spectrum between series $x(t)$ and $y(t)$ is estimated by

$S_{x y}(f)=\sum_{k=0}^{K-1} d_{x, k}(f) X_{k}(f) d_{y, k} Y_{k}^{*}(f)$

where $X_{k}$ and $Y_{k}$ are the eigenspectra of the $k$-th taper given in Eq. (1).

The phase of $S_{x y}(f)$ is in fact the phase difference between data series $x(t)$ and $y(t)$. If two data sets exhibit high phase coherency at a specific frequency it would be expected that the phase difference would be approximately constant at this frequency for the duration of the pulsation activity. The phase differences between the $2.1 \mathrm{mHz}$ multitaper analytic signals of ACE $\boldsymbol{v}_{x}$ (blue) and $\boldsymbol{v}_{y}$ (red) and the radar Doppler velocity are plotted in the lowest panel of Fig. 8. Shaded bands highlight intervals when $2.1 \mathrm{mHz}$ pulsation ac-

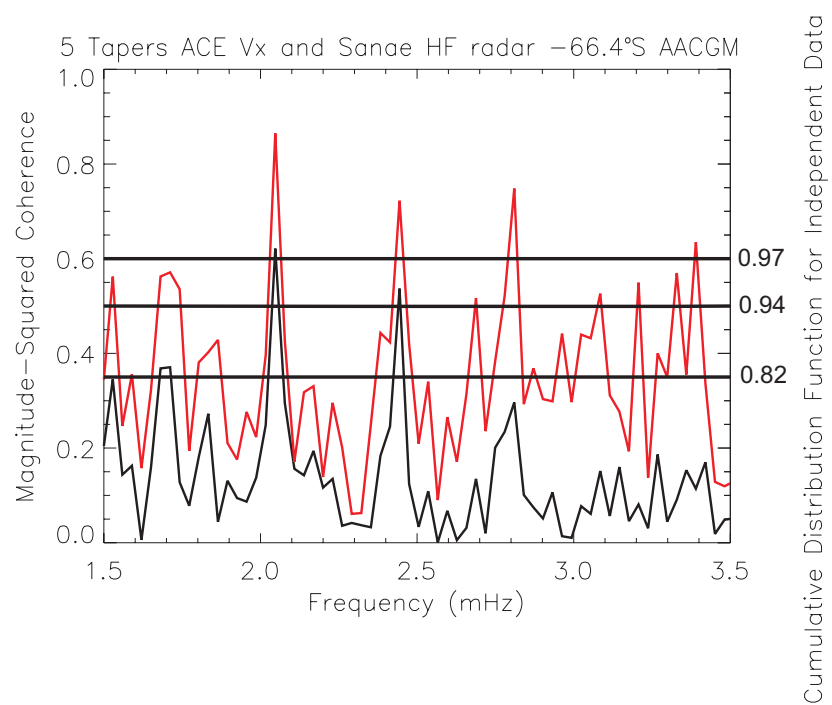

Fig. 10. $A C E V x$ and $H F$ radar coherence estimates. The black curve is the MSC and the red curve is one standard deviation above it. Left hand axis shows MSC on a linearized scale. The right had side is the CDF, an estimate of the actual probability levels.

tivity maximizes. Taking into account that the data were time shifted to compensate for travel time from the ACE satellite to the magnetosphere, these periods are coincidental. During these periods the phase difference between the $\boldsymbol{v}_{x}$ and $\boldsymbol{v}_{y}$ ACE velocity components and the radar is relatively constant, implying that they are locked with respect to one another in phase. Analyzing the data sets using a multiple window approach reveals the phase difference for the duration of the event, unlike unique windowing which can only reveal pulsation activity in the middle body of the event. In addition, it allows the phase difference to be evaluated at a high temporal resolution.

An alternative way to demonstrate phase coherence is to present it in the form of a spectrum, as depicted in Fig. 9. The cross-phase, determined from a cross-correlation of multitaper eigenspectra of ACE $V_{\mathrm{x}}$ and Radar Doppler velocity, is plotted against frequency. In this case, the data set was shortened to 512 samples, covering the period of pulsation activity. If the intervals where the $2.1 \mathrm{mHz}$ signal was absent were included, it would obviously weaken the coherency. Curtailing the data set in this way affects the frequency resolution of the spectrum and so a new choice of the time bandwidth product of $N W \triangle t=3$ was found to be appropriate, and $K=5$ tapers were applied. One standard deviation of the cross-phase also is plotted in red. This is narrow near the resonances of 1.9 and $2.1 \mathrm{mHz}$ illustrating low variability. The standard deviation was calculated in the following way. Non parametric estimates of the variability of the cross-phase were determined by jackknifing, a technique that is well accepted. The multitaper spectrum is calculated by omitting 


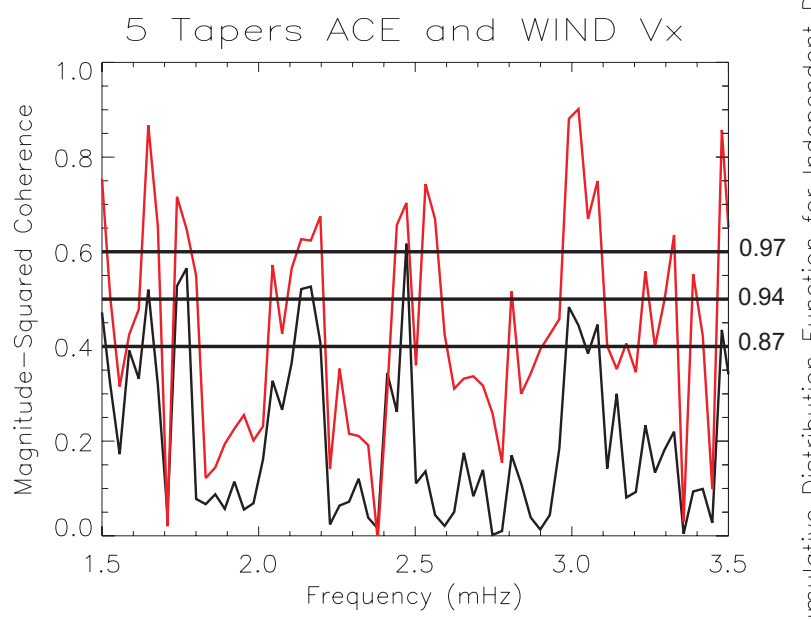

Fig. 11. MSC between ACE and WIND Vx components. Axes and curves same as Fig. 10.

each of the $K$ tapers in turn (Kuo et al., 1990; Thomson and Chave, 1991). Let $v_{-j}$ denote the coherency calculated with window $k=j$ omitted in Eqs. (2) and (6) one can compute an average $v$. such that

$v .=\frac{1}{K} \sum_{k=0}^{K-1} v_{-j}$

And variance

$V=\frac{K-1}{K} \sum_{k=0}^{K-1}\left|v_{-j}-v \cdot\right|^{2}$

The factor $K-1$ occurs as the estimates of $v_{-j}$ are correlated (Thomson and Chave, 1991). The jackknifed standard deviation shown as red lines in Figs. 9, 10 and 11 is simply $\sqrt{V}$. This method of determining standard deviation is known to work for a wide range of distributions, essentially all distributions for which maximum-likelihood estimates exist (Reeds, 1978) and is simpler to calculate than other distributions.

In addition, the amplitude coherency can be calculated i.e.

$C_{x, y}(f)=\frac{S_{x y}(f)}{\sqrt{ }\left(S_{x}(f) \cdot S_{y}(f)\right)}$

The scales of the coherence plots in Figs. 10 and 11 indicate the degree of coherency between the two series. The left axis shows Magnitude Squared Coherence (MSC), $\left|C_{x, y}(f)\right|^{2}$ on a linearized scale, analogous to a correlation coefficient in the time domain. In order to obtain a quantitative interpretation of the coherence, however, the result must be compared to, for example, the coherence between two independent series. In such a case, the probability that the MSC would be less than some quantity, say $u$, is:

$$
P(\mathrm{MSC} \leq u)=1-(1-u)^{K-1}
$$

where $K$ is the number of windows. This formula is the basis for calculating the Cumulative Distribution Function (CDF), an estimate of actual probability levels, shown on the right axis of Figs. 10 and 11. For example, in Fig. 10 the largest peaks are near 2.1 and $2.5 \mathrm{mHz}$. The $2.1 \mathrm{mHz}$ peak has a $\mathrm{CDF}$ of 0.97 or greater. This means that there is $97 \%$ probability that the spectral power of ACE $V_{\mathrm{x}}$ component and the $\mathrm{HF}$ radar Doppler velocity at $\left(-66.4^{\circ} \mathrm{S}\right)$ are correlated in this frequency band. This peak was the most significant $(99 \%)$ in Fig. 6 showing the multitaper spectrum of radar Doppler velocity stack plot. Its coincidence with significant peaks in ACE spectra was highlighted by shaded panels in Fig. 5. In Fig. 11 the MSC between the $V_{\mathrm{x}}$ components of ACE and WIND data is plotted. These satellites were some $180 R_{\mathrm{E}}$ apart and yet there exist very high levels of coherence for a number of frequency bands. In particular, a coherence of better than $94 \%$ is achieved for the $2.1 \mathrm{mHz}$ band.

\section{Discussion}

The purpose of this paper is to investigate whether or not coherent oscillations in the solar wind can give rise to Pc5 field line resonances within the magnetosphere at the same frequency. Even the existence of coherent oscillations in the solar wind has been a subject of controversy in the pulsation community. We believe that the fact of their occurrence should not be controversial. While some of the analyses of solar wind data have been open to criticism we consider that the work of Thomson et al. $(2001,2002)$; Ghosch et al. (2009) shows their existence beyond reasonable doubt. What is controversial is whether such oscillations have any relevance to the generation of Pc5 pulsations in the magnetosphere at the same frequency. We are not making the case that they are the only source of such pulsations. Nor are we making the case that they are the most important source. What we are seeking to show is that they are a source that cannot be neglected when seeking the cause of such events.

The paper is an event study and we have chosen to present a detailed investigation of one event while noting that several others that have been identified show similar features. Thus nothing we say has any bearing on the relative importance of different sources.

The multitaper spectra that we have presented provide unambiguous evidence that there is coherent narrow band spectral power in the $2.1 \mathrm{mHz}$ band at the locations of ACE, WIND, and the Sanae radar. There is similar evidence for other events for which we have not presented a detailed analysis. For this event in other frequency bands there is also power at a significance level that is not as high as for the $2.1 \mathrm{mHz}$ band. In Fig. 5 peaks occur at better than the 95\% confidence level at $1.9 \mathrm{mHz}$ (and $2.1 \mathrm{mHz}$ ) in all (and 5 of 8) the components observed by ACE, and in Doppler velocity range gate 15, beam 4 of the radar, while in Fig. 6 the same is true for a number of adjacent range gates of the Sanae radar. 
Any oscillation in the solar wind at these frequencies must correspond to an MHD wave. The candidates are the transverse Alfvén wave, the fast and slow magnetosonic waves, and the so-called entropy wave (Landau et al., 1984). Both fast and slow waves are Landau damped in a thermal plasma, the slow wave extremely heavily. Before excluding them as candidates it should be noted that the solar wind is far from thermal equilibrium and Landau growth is also possible in the right circumstances. In any event, for our purposes, it is unnecessary to identify the type of wave. The transverse Alfvén wave travels with the Alfvén velocity in the rest frame of the solar wind. The fast and slow waves travel with a hybrid speed between the Alfvén and sound speeds. The entropy wave is fixed in the solar wind. For all typical circumstances these wave speeds are much less than the solar wind speed in the Sun-Earth frame. Thus, if we observe any such wave in the Sun-Earth frame its velocity will not differ substantially from the solar wind velocity. On this occasion during the event the solar wind velocity was about $500 \mathrm{~km} \mathrm{~s}^{-1}$, while the characteristic wave speeds in the rest frame are about $10 \%$ of that. A $2.1 \mathrm{mHz}$ signal has a period of $480 \mathrm{~s}$ (about $8 \mathrm{~min}$ ). The wavelength of the oscillation is therefore about $240000 \mathrm{~km}$ or $38 R_{\mathrm{E}}$. ACE is $240 R_{\mathrm{E}}$ upstream and WIND well to one side of the Sun-Earth line. The distance between them is about $200 R_{\mathrm{E}}$, or roughly 5 wavelengths. We are thus providing evidence of narrow band oscillations over an extended period in the same frequency band at two positions in the solar wind that are separated by 5 wavelengths. Power in the same band is observed by the radar which is $240 R_{\mathrm{E}}$ (6 wavelengths) from ACE and $70 R_{\mathrm{E}}$ ( 2 wavelengths) from WIND.

Figure 7 relates to the reconstituted signal in the $2.1 \mathrm{mHz}$ band. In Fig. 7b, because the time series found from the complex demodulation of the multitaper spectrum gives a good estimate of the signal over the whole time series, we see that the event at the radar occurs in two bursts between 620 and $860 \mathrm{~min}$ (taking into account 57 min delay time) after the arbitary time origin at 01:31 UT. There is power in the frequency band throughout the time interval but, of course, this is the filtered broad band power arising from the turbulence, and gives an idea of how much stronger the coherent oscillation is than the turbulent background.

Figure 8a shows the energy flux calculated at ACE. The shaded regions are the time intervals during which strong oscillations occur. It shows the same two bursts as are seen shifted in time by the propagation time between spacecraft and radar. It shows the same double burst structure as seen at the radar $57 \mathrm{~min}$ later. There is an interesting feature, however. The first burst is associated with a strong oscillation in $V_{\mathrm{x}}$ and $V_{\mathrm{y}}$. During the second burst the oscillation is almost absent in the $V_{\mathrm{x}}$ component. We speculate that the two bursts might have different polarizations suggesting a different propagation mode in each case. This needs further analysis. Figure $8 \mathrm{~b}$ shows the power per unit area transferred to the ionosphere by Joule heating. Estimates of the total energy falling on the front of the magnetosphere show that it is more than sufficient to supply the energy deposited in the ionosphere. This does not exclude the possibility of amplification at the bow shock (McKenzie, 1970) or at the magnetopause (Mann et al., 1999).

So far we have shown strong evidence for the existence of coherent oscillations in the solar wind that coincide with field line resonances at the same frequency at the radar, delayed by the propagation time. To evaluate the possibility of a coincidence we have also performed a coherence analysis. The maximum squared coherence plotted in Figs. 10 and 11, show how significant this is. In the $2.1 \mathrm{mHz}$ band the coherence between ACE $V_{\mathrm{x}}$ and Sanae Doppler velocity is significant at better than the $97 \%$ confidence level. That between ACE and WIND is significant at very much better than the $94 \%$ confidence level. The lower coherence between in the $1.9 \mathrm{mHz}$ band is because, in this analysis, the data series has been shortened to specifically cover the interval of $2.1 \mathrm{mHz}$ resonance occurrence.

While all these results are compelling, it is in Figs. 8c and 9 that the significance of the relationship becomes most apparent. Figure $8 \mathrm{c}$ shows the phase difference between the reconstituted signals at ACE and Sanae in the time domain. If these signals at the same frequency have occurred by chance then their phase difference should vary randomly with time. This is the case over most of the period but, during the two burst of activities the phase difference between spacecraft and radar remains approximately constant; the two signals are phase locked strongly suggesting a causal relationship. Figure 9 shows the same effect in the frequency domain. The cross-phase represents the phase difference between the two signals as a function of frequency. As is to be expected this varies throughout the frequency range. However, the standard deviation at a particular frequency is a measure of the amount by which the phase difference varies over the time interval. Over most frequencies the standard deviation is large, sometimes many radians showing that the phase difference at that frequency varies substantially with time. Within the two frequency bands of interest, however, the standard deviation is a small fraction of a radian showing that the oscillations in those bands are dominated by signals that are locked together in phase. This we regard as very strong evidence for a causal relationship between the signal seen at the spacecraft and that in the ionosphere.

We have presented a detailed analysis of an event that shows very strong correlation between the Pc5 frequency oscillations seen at both WIND and ACE and those seen at the appropriate delay time at the Sanae radar. We consider the evidence of a causal relationship to be very strong. We have identified a few similar events, but at present cannot estimate the relative importance of this mechanism and other mechanisms that might cause field line resonance. 


\section{Conclusions}

1. On 7 June 2000 the magnetic field and plasma instruments on ACE and WIND observed two intensifications of coherent oscillations in the Pc5 band, delayed appropriately according to the spacecraft separation. These oscillations in the 1.9 and $2.1 \mathrm{mHz}$ bands were significant at better than the $95 \%$ confidence level.

2. The SuperDARN radar SHARE at Sanae Antarctica showed similar bursts of activity in the Doppler velocity, delayed appropriately from the spacecraft observations. These showed the features of field line resonances at the same two frequencies, also significant at better than the $95 \%$ level.

3. The signals between each pair of instruments were correlated and showed a maximum squared coherence that was significant at better than the $94 \%$ confidence level.

4. The standard deviation of the cross phase between the instruments was small at the location of the peaks in the spectrum and large elsewhere where the spectrum was essentially due to noise.

5. Signals reconstructed by complex demodulation at ACE, WIND, and SHARE showed approximately constant phase difference during the bursts of activity. At other times the phase difference varied randomly.

6. The multiple taper method provided a superior means of analysing the data, giving objective estimates of the confidence limits and sampling the time series uniformly throughout the seventeen hour data set.

This leads us to conclude that, on this occasion, and on two others showing similar features, coherent oscillations in the solar wind directly drove field line resonances at the same frequencies in the magnetosphere.

The evidence does not allow us to evaluate the relative importance of this mechanism and other sources of field line resonance.

Acknowledgements. The SHARE HF radar at Sanae, Antarctica is supported by funding from National Research Foundation of South Africa (NRF) and the Antarctic logistics are provided by the South African Department of Environment Affairs and Tourism (DEAT). We thank WIND and ACE instrument and science teams of the MFI, SWEPAM and SWE instruments for providing the solar wind data. We thank members of the SSA-MTM team of the Department of Atmospheric Sciences, UCLA and US Geological Survey and the Commissariat à l'Energie Atomique as well as all other individuals responsible for the development and maintenance of the Toolkit used in the multitaper analysis presented here.

Topical Editor I. A. Daglis thanks one anonymous referee for her/his help in evaluating this paper.

\section{References}

Baker G., Donovan, E. F., and Jackel, B. J.: A comprehensive survey of auroral latitude Pc5 pulsation characteristics, J. Geophys. Res., 108(A10), 1384, doi:10.1029/2002JA009801, 2003.

Barnes, A.: Hydromagnetic waves and turbulence in the solar wind, in: Solar System Plasma Physics, Vol 1 Solar and Solar Wind Plasma Physics, edited by: Parker, E. N., Kennel, C. F., and Lanzerotti, L. J., North-Holland, New York, 249-319, 1979.

Bracewell, R. N.: The Fourier Transform and its applications, 2nd ed., 268, McGraw-Hill, New York, 1986.

Brillinger, D. R.: Time series: Data analysis and theory (expanded edition), Holden-Day, San Francisco, 1981.

Chisham, G., Lester, M., Milan, S. E., Freeman, M. P., Bristow, W. A., Grocott, A., McWilliams, K. A., Ruohoniemi, J. M., Yeoman, T. K., Dyson, P. L., Greenwald, R. A., Kikuchi, T., Pinnock, M., Rash, J. P. S.,Sato, N., Sofko, G. J., Villain, J.P., and Walker, A. D. M.: A decade of the Super Dual Auroral radar Network (SuperDARN): scientific achievements, new techniques and future directions, Surv. Geophys., 28, 33-109, doi:10.1007/s10712-007-9017-8, 2007.

Dettinger. M. D., Ghil, M., Strong, C. M., Weibel, W., and Yiou, P.: Software expedites singular-spectrum analysis of noisy time series, Eos, Trans. American Geophys. Union, v.76(2), 11, 1421, 1995.

Dudeney, J. R., Baker, K. B., Stoker, P. H., and Walker, A. D. M.: The Southern Hemisphere Auroral Radar Experiment (SHARE), Antarctic Sci., 6, 123-124, 1994.

Eriksson, P. T. I., Walker, A. D. M., and Stephenson, J. A. E.: A statistical correlation of Pc5 pulsations and solar wind pressure oscillations, Adv. Space Res., 38, 1763-1771, doi:10.1016/j.asr.2005.08.023, 2005.

Fenrich, F. R. and Samson, J. C.: ULF high- and low- $m$ field line resonances observed with the Super Dual Auroral Radar Network, J. Geophys. Res., 100(A11), 21535-21547, 1995.

Fenrich, F. R. and Waters C. L.: Phase coherence analysis of a field line resonance and solar wind oscillation, Geophys. Res. Lett., 35, L21002, doi:10.1029/2008GL035430, 2008.

Ghil, M., Allen, M. R., Dettinger, M. D., Ide, K., Kondrashov, D., Mann, M. E., Robertson, A. W., Saunders, A., Tian, Y., Varadi, F., and Yiou, P.: Advanced spectral methods for climatic time series, Rev. Geophys., 40(1), 3.1-3.41, doi:10.1029/2001RG000092, 1995.

Ghosch, S., Thomson, D. J., Matthaeus, W. H., and Lanzerotti, L. J.: Coexistence of turbulence and discrete modes in the solar wind, J. Geophys. Res., 114, A08106, doi:10.1029/2009JA014092, 2009.

Greenwald, R. A. and Walker, A. D. M.: Energetics of long period resonant hydromagnetic waves, Geophys. Res. Lett., 7, 745-748, 1980.

Kepko, L., Spence, H. E., and Singer, H. J.: ULF waves in the solar wind as direct drivers of magnetospheric pulsations, Geophys. Res. Lett., 29(8), 39-1-4, doi:10.1029/2001GL014405, 2002.

Kivelson, M. G. and Southwood, D. J.: Resonant ULF waves: A new interpretation, Geophys. Res. Lett., 12, 49-52, 1985.

Kuo, C., Lindberg, C., and Thomson, D. J.: Coherence established between atmospheric carbon dioxide and global temperature, Nature, 343, 709-714, 1990.

Landau, L. D., Pitaevskii, L. P., and Lifshitz, E. M.: Electrodynamics of Continuous Media, 2nd edition, Pergamon Press, Oxford, 
1984.

Lepping, R. P., Acuña, M. H., Burlaga, L. F., Farrell, W. M., Slavin, J. A., Schatten, K. H., Mariani, F., Ness N. F., Neubauer, F. M., Whang, Y. C., Byrnes, J. B., Kennon, R. S., Panetta, P. V., Scheifele, J., and Worley, E. M.: The Wind magnetic field investigation, Space Sci. Rev, 71, 207-229, 1995.

Libbrecht, K. G., Woodward, M. F., and Kaufman, J. M.: Frequencies of solar oscillations, Astrophys. J. Suppl. Series, 74, 11291149, 1990.

Mann, I. R., Wright, A. N., Mills, K. J., and Nakariakov, V. M.: Excitation of magnetospheric waveguide modes by magnetosheath flows, J. Geophys. Res., 104(A9), 333-353, 1999.

McComas, D. J., Blame, S. J., Barker, P., Feldman, W. C., Phillips, J. L., Riley, P., and Griffee, J. W.: Solar Wind Electron Proton Alpha Monitor (SWEPAM) for the Advanced Composition Explorer, Space Sci. Rev., 86, 563-612, 1998.

McKenzie, J. F.: Hydromagnetic wave interaction with the magnetopause and the bow shock, Planet. Space Sci., 18, 1-23, 1970.

Ogilvie, K. W., Chornay, D. J., Fritzenreiter, R. J., Hunsaker, F., Keller, J., Lobell, J., Miller, G., Scudder, J. D., Sittler Jr., E. C., Torbert, R. B., Bodet, D., Needell, G., Lazarus, A. J., Steinberg, J. T., Tappan, J. H., Mavretic, A., and Gergin, E.: SWE, A comprehensive plasma instrument for the Wind spacecraft, Space Sci. Rev., 71, 55-77, 1995.

Percival, D. B. and Walden, A. T.: Spectral analysis for physical applications. Multitaper and conventional univariate techniques, Cambridge University Press, Cambridge, 1993.

Potemra, T. A., Lühr, H., Zanetti, L. J., Takahashi, K., Erlandson, R. E., Marklund, G. T., Block, L. P., Blomberg, L. G., and Lepping, R. P.: Multisatellite and ground-based observations of transient ULF waves, J. Geophys. Res., 94(A3), 2543-2554, 1989.

Prikryl, P., Greenwald, R. A., Sofko, G. J., Villian, J. P., Ziesolleck, W. S., and Friis-Christensen, E.: Solar-wind-driven pulsed magnetic reconnection at the dayside magnetopause, Pc5 compressional oscillations, and field line resonances, J. Geophys. Res., 103(A8), 17307-17322, 1998.

Prikryl, P., MacDougall, J. W., Grant, I. F., Steele, D. P., Sofko, G. J., and Greenwald, R. A.: Observations of polar patches generated by solar wind Alfvén wave coupling to the dayside magnetosphere, Ann. Geophys., 17, 463-489, 1999,

http://www.ann-geophys.net/17/463/1999/.

Reeds, J. A.: Jackknifing maximum likelihood estimates, Ann. stat., 6, 727-739, 1978.

Ruohoniemi, J. M., Greenwald, R. A., Baker, K. B., and Samson, J. C.: HF radar observations of Pc5 field line resonances in the midnight/early morning MLT sector, J. Geophys. Res., 96(A9), 15697-15710, 1991.

Samson, J. C., Harrold, B. G., Ruohoniemi, J. M., Greenwald, R. A., and Walker, A. D. M.: Field line resonances associated with MHD waveguides in the magnetosphere, Geophys. Res. Lett., 19, 441-444, 1992.
Shimazu, H., Araki, T., Kamei, T., and Hanado, H.: A symmetrical appearance of Pc5 on dawn and dusk sides associated with solar wind pressure enhancement, J. Geomagn. Geoelect., 47, 177189, 1995.

Sibeck, D. G.: Transient events in the outer magnetosphere: Boundary waves or flux transfer events?, J. Geophys. Res., 97(A4), 4009-4026, 1992.

Smith, C. W., Acuna, M. H., Burlaga, L. F., L'Heureux, J., Ness, N. F., and Scheifele, J.: The ACE Magnetic Fields Experiment, Space Sci. Rev., 86, 611-632, 1998.

Southwood, D. J.: The hydromagnetic instability of the magnetospheric boundary, Planet. Space Sci., 16, 587-605, 1968.

Stephenson, J. A. E. and Walker, A. D. M.: HF radar observations of Pc5 ULF pulsations driven by the solar wind, Geophys. Res. Lett., 29(9), 1297-1300, doi:10.1029/2001GL014291, 2002.

Thomson, D. J.: Spectrum Estimation and Harmonic Analysis, Proceedings of the IEEE, 70, 1055-1096, 1982.

Thomson, D. J. and Chave, A. D.: Jackknifed error estimates for spectra, coherences, and transfer functions, in: Advances in spectrum analysis and array processing, Vol. 1, edited by: Haykin, S., Englewood Cliffs, Prentice-Hall, New Jersey, 58-113, 1991.

Thomson, D. J., Maclennan, C. G., and Lanzerotti, L. J.: Propogation of solar oscillations through the interplanetary medium, Nature, 376, 139-144, 1995.

Thomson, D. J., Lanzerotti, L. J., and Maclennan, C. G.: Interplanetary magnetic field: Statistical properties and discrete modes, J. Geophys. Res., 106, 15941-15962, 2001.

Thomson, D. J., Lanzerotti, L. J., and Maclennan, C. G.: Studies of some statistics of the interplanetary magnetic field and implications for discrete modes, Adv. Space Res., 29(12), 1911-1916, 2002.

Walker, A. D. M., Ruohoniemi, J. M., Baker, K. B., and Greenwald, R. A.: Spatial and temporal behavior of ULF pulsations observed by the Goose Bay HF radar, J. Geophys. Res., 97(A4), 12187$12202,1992$.

Walker, A. D. M.: Reflection and transmission at the boundary between twocounterstreaming MHD plasmas - active boundaries or negative-energy waves?, J. Plasma Phys., 63, 203-219, 2000.

Walker, A. D. M.: Excitation of field line resonances by MHD waves originating in the solar wind, J. Geophys. Res., 107(A12), 1481, doi:10.1029/2001JA009188, 2002.

Walker, A. D. M.: Excitation of field line resonances by sources outside the magnetosphere, Ann. Geophys., 23, 3375-3388, 2005, http://www.ann-geophys.net/23/3375/2005/. 